\title{
Multivariate time Series Analysis of Neuroscience Data: Some Challenges and Opportunities
}

\author{
Mohsen Pourahmadi \\ Department of Statistics \\ Texas A\&M University \\ College Station, TX 77843-3143 \\ Siamak Noorbaloochi \\ VA HSR\&D Center for Chronic Diseases Outcome Research \\ and Department of Medicine \\ University of Minnesota \\ Minneapolis, MN 55455-0460
}

\begin{abstract}
Neuroimaging data may be viewed as high-dimensional multivariate time series, and analyzed using techniques from regression analysis, time series analysis and spatiotemporal analysis. We discuss issues related to data quality, model specification, estimation, interpretation, dimensionality and causality. Some recent research areas addressing aspects of some recurring challenges are introduced.
\end{abstract}




\section{Introduction}

There are myriad of challenges in the multivariate analysis of high-dimensional neuroimaging (fMRI) and neurophysiological data ( EEG, local field potential (LFP), and spiking activity). In this note we focus on fMRI data, although the same insights can be applied to other types of data. The basic characteristic of such data is that all are time series and indeed are sample paths of stochastic processes.

In fMRI data, the goal is to understand neural activity over time representing the hemodynamic response function of the brain, the possible connectivity between neurons, regions, and their relationship to behavior and stimuli. Data (signals) collected in neuroscience experiments are usually noisy, subject to periodic respiratory and cardiac noise and low frequency noise due to physiological shifts, movements and instrumental instabilities. Preprocessing [4] is an integral and fairly ad-hoc way of handling some of these unintended mishaps during an experiment which prepares the data for the next and more methodic phase of statistical or time series analysis. Preprocessing may reduce these unintended effects, and when done right one might end up with a dataset having considerable temporal and spatial correlations. Ignoring these correlations violates the basic assumptions of regression analysis or linear models and can give rise to serious statistical issues such as bias and incorrect significance level. Dealing with the correlations, however, leads to computational and other inferential challenges in practice, some of which are discussed in this overview. 


\section{Modeling Options for fMRI Data}

In this section the roles of simple linear regression, time series, and spatiotemporal models for the analysis of fMRI data are reviewed. These models are ordered from the simplest to the more complex based on their ability to incorporate the correlations in the data.

Data in neuroscience often contain enormous number of variables measured over time and space (surface of the brain) with the inherent temporal and spatial correlations. For example, even after extensive preprocessing and downplaying spatial correlations, fMRI data may be viewed as a multivariate (stationary) time series where the signal from each voxel is simply a univariate series. However, the number of voxels could be hundreds of thousands ([5], [6]) giving rise to a high-dimensional time series. Although, many other organisms studied by neuroscientists have less neurons and less connections, with over 86 billion of neurons and $\sim 1000$ potential connections per neuron in the human brain, statistical modeling becomes a real challenge both from computational and inferential perspectives. The presence of confounders and differential neural responses to the stimuli and possible patterns of various time-varying behaviors further complicates development of models producing plausible inferences.

The same issues may be present even in problems with much smaller neural populations.

In spite of these issues, there has been considerable progress in incorporating temporal correlations in statistical analysis of fMRI data. Dimension reduction techniques such as principal component analysis, canonical correlations, partial least 
square, and independent component analysis are powerful tools for the analysis of fMRI data. However, often the summaries may ignore or distort the intrinsic temporal dependence in the data.

The journey from univariate regression analysis to full spatiotemporal analysis, and the happy medium offered by time series analyses, display the indispensable challenges one is faced with in ensuring relevancy of statistical inference to the reality. Regression Analysis: Univariate modeling completely ignores the temporal and spatial correlations, identification of active voxels amounts to analyzing one voxel at a time. There are no computational issues due to the univariate nature of the problem, however, there are serious inferential issues related to assessing the Type I error due to ignoring the correlations, for more details see [2, Chap. 5].

Time Series Analysis: The main distinction of multivariate series from the standard multivariate data is the temporal-dependence in the former which carries considerable information about the underlying phenomenon of interest. There are two general approaches to analyzing time series data where both are concerned with decorrelating or whitening the data using conceptually different tools. These are the time-domain and frequency-domain approaches where the former relies on the covariance or correlation of the data and the latter on the Fourier transform. Traditionally, the former has been popular in economics and social sciences where the sample sizes are smaller, and the notion of correlation is more tangible. A notion of stationarity (time-invariance of moments of up to order two) is central to both approaches to time series analysis. 
The simplest way to think of the relevance of the time-domain approach to fMRI data is to ignore the spatial correlations among the voxels and use the standard finite parameter autoregressive and moving average (ARMA) models for the time course data for each voxel separately. An autoregressive (AR) model essentially is the regression of the current observation in terms of the previous observations and errors. A movingaverage (MA) model is a linear regression of the current value of the series against current and previous (unobserved) independent error terms or random shocks typically coming from a normal distribution, with mean zero and constant variance.

There is a strong preference for pure AR models which are seen as analogues of regression models and the framework is adapted rather easily to several voxels giving rise to the popular vector AR (VAR) models in the fMRI literature. This class of models is ideal for testing causality and directions of connectivity among several regions of the brain.

Spatiotemporal and Spatial Analysis: Ideally, one should rely on spatiotemporal models for fMRI data where both temporal and spatial correlations are employed in the analysis. However, formulating genuinely realistic and statistically valid spatiotemporal models that capture both sources of correlations has encountered numerous computational and conceptual challenges hampering progress in this rather natural direction. It should be noted that standard spatial models which relate correlations to distance of locations in space may not be suitable for fMRI data since voxels in different hemispheres of the 
brain, though far away, are known to be highly correlated. The good news is that given a reasonable temporal, and spatial correlation model, as a first approximation, one can form separable spatiotemporal models [2, p. 127].

\section{Graphical Models}

Graphical models are powerful tools for displaying correlations and possibly directions of influence among several variables. In this section, the roles of Gaussian graphical models and multiple graphs in the analysis of fMRI data are reviewed.

The goal of modeling brain connectivity is achieved by constructing a graph where its vertices represent different voxels (locations) while an edge between two vertices reflect a connection between the two voxels. Graphs are of fundamental importance in the brain connectivity studies which have the goal of understanding higher human brain functions (networks) via the association among the spatially remote neurons in various brain regions. For example, the functional connectivity is concerned with the undirected correlation between spatially remote neurophysiological events and the effective connectivity deals with directed or causal relations among brain regions [7]. The absence of an edge is indicated by a null partial coherence for the two signals, and the ability to detect it is the key in the construction of a meaningful graph. Note that coherence at each frequency is a measure of linear dependence between the two signals at that frequency. The common method to estimate the coherency function is the Welch method which by segmenting the signals as a function of frequencies uses the Pearson correlation formula to construct the estimate for each segment. Partial coherence in each frequency is the partial correlation between the signals adjusted for 
other signals at the given frequency. Tests of significance of coherency based on Gaussian model [8], or Monte Carlo methods [9] are available. However, usually eyeballing the (partial) coherence function is essential to gain insights about the coherency.

In multivariate statistics the notion of Gaussian graphical models has received considerable attention in recent years where estimation of the high-dimensional inverse covariance (precision matrix) and the notion of partial correlations are the key ingredients. An important reason for the popularity of Gaussian graphical models is the simple observation that conditional independence is equivalent to partial correlations being zero, and entries of the precision matrix are proportional to the partial correlations. In particular, regression interpretation of entries of the precision matrix has led to a host of fast methods of computing the sparse precision matrix, referred to as the graphical LASSO (GLASSO) methods. These methods rely on independent observations and penalized likelihood method in search of sparse and interpretable graphs.

The Key Ingredient: In spectral analysis of multivariate time series, the spectral density matrix plays the role of the covariance matrix, from which on can compute the inverse spectral density matrix. Partial coherence, the spectral-domain analogue of the partial correlation, is the key measure of functional connectivity which identifies the frequency region that drives the correlation between any two voxels (component series) adjusted for the linear effects of other voxels. However, at present, there is a problem with the 
estimation of the partial coherences. Surprisingly, all existing methods ([10]; [11]) estimate the spectral density matrix first, and then compute its inverse at the Fourier frequencies. As expected, these methods work well both computationally and statistically when the number of series (voxels) is small or moderate, but will suffer from serious computational complexity and statistical instability problems when the dimension is large relative to the length of the series.

Joint or Multiple Graphs: Traditionally, Gaussian graphical models are concerned with estimating a single graph using independent realizations of a common random vector. Such a model is not adequate when data are collected from many individuals on the same set of variables having different dependence or covariance structures over time or other relevant covariates. For example, brain connectivity networks corresponding to different subjects vary, but are expected to be more similar if the corresponding subjects share common demographic such as age. In this setting, estimating the graphical models separately for each subject ignores the similarity between the adjacent graphs, while estimating one population graphical model on the basis of the data of all subjects ignores the differences between graphs. As a specific motivation for multiple graphs, consider a resting state fMRI data, where subjects are naturally ordered by their ages and the observations of each subject are multiple brain scans with temporal dependence. For such data, a non-parametric kernel-based estimation approach which efficiently estimates and predicts multiple networks is proposed in [12]. Interestingly, the underlying model in the proposed approach can be viewed as spatiotemporal models indexed by covariates. 


\section{Causality}

The elusive notion of causality or directed influence is discussed in this section. A plausible and estimable notion of causal effect of one process (intervention, stimuli, and concomitant covariates) on the observed response neurons (or voxels) activities has been a great challenge. There are at least four notions of causality for multivariate time series [13]. The most popular and classical notion of causality is the Granger causality [14] initially developed in the econometrics literature.

For linear vector autoregressive (VAR) models one may develop a Granger causal graph by testing whether the VAR coefficients and partial correlations between stimuli and the targeted responses are zero. There are at least two issues with such a plan: First, the estimates of the coefficients depend on what we have in the model and hence non-causality decision depends on the covariates in the model and not on the intrinsic possible causality between the "effect process" and the response signal. This issue is present in regression models: when $X$ is used to predict $Y$ and one is going to use the estimated coefficient of $X$ as a measure of importance of $X$, by varying inclusion of other moderating predictors in the model, one might find quite different estimates for the coefficient of X. Due to lack of an exhaustive list of concomitant covariates, and, the possible existence of influential omitted variables, any causal argument based on such estimates should be viewed with some qualification. Secondly, the dimensionality reduction of different processes, which depending on their role in the model, may need different treatment. The dimension-reduction of the confounding (blurring) processes 
can be achieved using, say, partial least square or canonical correlation, while the dimension-reduction of the response processes, in multi-neuron/multi voxels may be based on the connectivity structure one may have hypothesized between different activated neurons. For example, in reducing the dimensionality of covariate space, a principal component extraction of the predictors might have a different dependence structure with the targeted response than the original variables, making it less desirable for studying connectivity issues, while the same method, might be very useful when a general index of the multiple responses is of prime interest. In such situations, a hypothesized graphically represented structure is very valuable for modelling the complex system. The Granger causal graphs for example, are good visual tools to depict and study the relationships between the involved subprocesses [15]. For a recent lucid introduction to Granger causality see [16].

\section{Conclusions}

Preprocessed fMRI data can be modeled ideally using a multivariate regression model of the form $Y=X B+E$ with standard meaning attached to the four components of the model. The computational and statistical challenges cited earlier stem from either ignoring or trying to genuinely model the correlations in the error term E. Fortunately, multivariate time series analysis provides a happy medium between the two extremes of regression analysis which ignores the correlations and spatiotemporal models which aim at full-edged modeling of the correlations. 


\section{Acknowledgment}

The work of the first author was supported by the NSF grant DMS-1309586 and the second author was supported by IIR 12-340 HSR\&D grant, Department of Veterans Affairs, Veterans Health Administration, Office of Research and Development, Health Services Research and Development. The views expressed in this article are those of the authors and do not necessarily reflect the position or policy of the Department of Veterans Affairs or the United States government.

\section{References}

[1] K. Worsley, K. Friston, Analysis of fmri time-series revisited--again. Neuroimage 2 (1995) 173-181.

[2] N. Lazar, The Statistical Analysis of Functional MRI Data, Springer, New York, 2008.

[3] M. Lindquist, The statistical analysis of fmri data., Stat. Sci. 23 (2008) 439-464.

[4] J. Ashburner, K. Friston, in: Human Brain Function, 2nd Edition, Academic Press, 2003.

[5] T. Ozaki, Time Series Modeling of Neuroscience Data, Chapman \& Hall/CRC, New York, 2013. 
[6] S. Lee, H. Shen, Y. Truong, M. Lewis, X. Huang, Independent component analysis involving autocorrelated sources with an application to functional magnetic resonance imaging, J. of the Amer. Statist. Assoc. 106 (2011) 1009-1024.

[7] M. Eichler, A graphical approach for evaluating effective connectivity in neural systems, Philos. Trans. Roy. Soc. B 360 (2005) 953-967.

[8] G. Clifford Carter. Coherence and time delay estimation. Proceedings of the IEEE, 75(2):236-255,1987.

[9] S. Makeig and H. Inlow. Lapses in alertness: Coherence of fluctuations in performance and EEG spectrum. Electroencephalography and Clinical Neurophysiology, 86:23-35, 1993.

[10] R. Dahlhaus, Graphical interaction models for multivariate time series., Metrika 51 (2000) 157-172.

[11] M. Fiecas, H. Ombao, The generalized shrinkage estimator for the analysis of functional connectivity of brain signals, Ann. Appl. Stat. 5 (2A)(2011) 1102-1125. $[12]^{\star \star}$ H. Qiu, F. Han, H. Liu, B. Caffo, Joint estimation of multiple graphical models from high dimensional time series, J. R. Statist. Soc. B (Online: 2015) 1-18. 
The problem of jointly estimating multiple graphical models in high dimensions is studied using a kernel based method. They apply the method to real resting state functional magnetic resonance imaging (rs-fMRI) data to illustrate the effectiveness of the method.

$[13]^{\star}$ M. Eichler, Causal inference with multiple time series: principles and problems, Philos. Trans. Roy. Soc. A mathematical physical and engineering 371 (2013) 953-967.

It gives a theoretical justification relating Granger causality to other causality notions and outlines problems with spurious causality and how to tackle them. It also provides a non-technical account of an identification algorithm that learns causal time-series structures in the presence of latent variables.

[14] W. Granger, Investigating causal relations by econometric models and crossspectral methods, Econometrica 37 (1969) 424-438.

[15] M. Eichler, Causal inference in time series analysis, in: Causality: statistical prepectives and applications, 2nd Edition, Wiley, 2012, 327-354.

$[16]^{\star}$ A. K. Seth, A. Barrett, L. Barnett, Granger causality analysis in neuroscience and neuroimaging, The Journal of Neuroscience 35 (2015) 3293-3297.

It is a brief introduction to G-causality, its theoretical basis, computational strategy, and the possibilities and limitations for neuroscientists. 